\title{
The many faces of psoriatic arthritis - a challenge to treatment to target?
}

\author{
Daniel Aletaha \\ Division of Rheumatology, Medical University of Vienna, Vienna, Austria
}

Psoriatic arthritis (PsA) is a multi-faceted disease. It may involve not only the joints in the sense of arthritis, but also the tendons surrounding the joints, leading to swelling of whole digits (dactylitis), or it may lead to inflammation of the entheses (enthesitis). PsA is considered one of the seronegative spondyloarthropathies, because it may also affect the axial system (spondylarthritis), and it is obviously characterised by the co-presence of psoriatic skin lesions [1]. In addition, PsA is accompanied by a systemic inflammatory reaction, which can be assessed by acute phase measures (e.g., C-reactive protein - CRP) or the sedimentation rate. This inflammatory activity may stem from involvement of any of the organs mentioned above, and may be considered a summation of all systemic inflammatory events of PsA. Finally, and very importantly, patients with PsA suffer from considerable functional loss and impairment of quality of life [2].

Many treatments are now available for management of PSA, including methotrexate (MTX) and other conventional disease-modifying antirheumatic drugs (DMARDs), as well as numerous biological compounds [3]. Interestingly, the response to these compounds differs across the different manifestations of PsA: while MTX is effective for peripheral joint and skin disease, it is not, or at least much less, helpful for axial disease, enthesitis, or dactylitis; while TNF inhibitors appear to act on all manifestations of PsA in a substantial and quite comparable way, other treatment modalities, such as IL-12/IL-23 or apremilast, appear to work more on the skin and less on the joints. All these specifics of PSA are recognised in national and international management recommendations for this disease, such as those provided and updated by the European League Against Rheumatism (EULAR) [4].

All these differences have led to the belief that PSA is not a homogeneous disease, neither clinically, nor in its therapeutic amenability. While this heterogeneity of
PsA is widely appreciated, at the same time it is often - and quite in good faith - ignored when it comes to disease activity assessment: claims to combine assessment of all domains into single scores may - although seemingly practical at first sight - be counterproductive for several reasons. One reason is the ambiguity of the outcome measure that include domains with differential responsiveness to different treatments, as it will be, for example, difficult to discern whether a patient did not respond to treatment, or simply did not respond in all domains of such a "lumped" index. An other reason is partly linked to it: the low responsiveness of some domains may relate to the biology of the disease, e.g. different pathomechanisms of axial as opposed to peripheral joint disease, but may also be a consequence of the lower prevalence of the respective organ involvement.

Particularly, if one expands the treat-to-target concept from RA to PSA [5], it becomes apparent that specific instruments are required that reliably reflect the target. In other words, when we treat PsA to target, we may want to look at the skin separately, and differentiate between joint and skin manifestations. It is conceivable that different responses of different target organs may have different therapeutic implications.

In that regard, recently the Disease Activity Score for Psoriatic Arthritis (DAPSA) was developed and validated [6]. It has been shown that the use of composite scores is superior to the evaluation of individual variables by capturing several items and minimising between-patient and within-patient variability over time [7]. The DAPSA includes tender and swollen joints (TJC68, SJC66), the patient global scale and pain score (each on a $10 \mathrm{~cm}$ visual analogue scale), as well as CRP. The DAPSA therefore simply and purposely ignored - for the reasons mentioned above - the claim of some to be inclusive of all possible domains of PsA; it focussed on disease activity of psoriatic arthritis, which would be the target for

Address for correspondence:

Daniel Aletaha, Division of Rheumatology, Medical University of Vienna, Vienna, Austria, e-mail: daniel.aletaha@meduniwien.ac.at Submitted: 17.02.2016, Accepted: 22.02.2016 
treat to target of PsA. One common misunderstanding is that this does not preclude a separate assessment of other domains, such as enthesitis, dactylitis, or spinal involvement. It is even the opposite: all these domains must be assessed in patients who present with respective manifestations, and many organ-specific instruments have been developed for that purpose. As an example, in a patient with dominant axial involvement, the BASDAI (Bath Ankylosing Spondylitis Disease Activity Index) may be considered as the basis for treatment decisions/adaptations, and not the DAPSA, which might happen to be remittive.

Finally, one advantage of the DAPSA is that it allows the assessment of the actual level of disease activity. This is an important difference to responder indices, such as the PSACR (Psoriatic Arthritis Response Criteria) or the ACR response criteria, particularly in times when treat-to-target is the key concept, i.e. asking the question whether a patient actually achieved a good state, regardless of response. Physicians managing patients with PSA are therefore quite challenged in not only understanding the complexity of the disease as such, but also to make the judgement of measuring the right thing the right way in the right patient. Otherwise, overtreatment and undertreatment, the most substantial concerns in management of chronic disease, can easily become clinically relevant discussion points.

The author declares no conflict of interest.

\section{References}

1. Gladman D, Antoni C, Mease P, et al. Psoriatic arthritis: epidemiology, clinical features, course, and outcome. Ann Rheum Dis 2005; 64 (suppl 2): ii14-ii17.

2. Gelfand JM, Gladman DD, Mease PJ, et al. Epidemiology of psoriatic arthritis in the population of the United States. J Am Acad Dermatol 2005; 53: 573.

3. Ramiro S, Smolen JS, Landewe R, et al. Pharmacological treatment of psoriatic arthritis: a systematic literature review for the 2015 update of the EULAR recommendations for the management of psoriatic arthritis. Ann Rheum Dis 2016; 75: 490-498.

4. Gossec L, Smolen JS, Ramiro S, et al. European League Against Rheumatism (EULAR) recommendations for the management of psoriatic arthritis with pharmacological therapies: 2015 update. Ann Rheum Dis 2016; 75: 499-510.

5. Smolen JS, Braun J, Dougados M, et al. Treating spondyloarthritis, including ankylosing spondylitis and psoriatic arthritis, to target: recommendations of an international task force. Ann Rheum Dis 2014; 73: 6-16.

6. Schoels M, Aletaha D, Funovits J, et al. Application of the DAREA/DAPSA score for assessment of disease activity in psoriatic arthritis. Ann Rheum Dis 2010; 69: 1441-1447.

7. Goldsmith $\mathrm{CH}$, Smythe HA, Helewa A. Interpretation and power of a pooled index. J Rheumatol 1993; 20: 575-578. 